Supporting Information for

\title{
Individual Roles of Peptides PGLa and Magainin 2 in Synergistic Membrane Poration
}

Wendong $\mathrm{Ma}^{\dagger}$, Shuqing Sun', Wenwen Li', Zhihong Zhang, Zhao Lin, Yu Xia, Bing Yuan ${ }^{*}$, and Kai Yang*

Center for Soft Condensed Matter Physics and Interdisciplinary Research \& School of Physical Science and Technology, Soochow University, Suzhou, 215006, P. R. China

Number of pages: 10

Number of figures: 10

Number of tables: 1

Table of Contents:

1. Experimental details

2. Supplementary images

3. Supplementary table 


\section{Experimental details}

\section{GUV leakage assay}

Fabrication of GUVs. GUVs were prepared following the conventional electro-formation method. Briefly, a solution of lipids (DOPC containing 0.1 mol\% RhB-PE for fluorescence labeling; $60 \mu \mathrm{L}$ $\times 2.0 \mathrm{mg} \mathrm{mL}^{-1}$ in chloroform) was deposited onto two ITO-coated glass slides and dried under vacuum overnight. The dry film was transferred in a homemade electro-formation chamber (with the two glass slides as electrodes), and rehydrated in $0.1 \mathrm{M}$ sucrose buffer. Alternating voltages were applied $(0.5 \mathrm{~V} \times 20 \mathrm{~min}, 1.0 \mathrm{~V} \times 20 \mathrm{~min}$ and $1.5 \mathrm{~V} \times 3 \mathrm{~h})$. The obtained vesicles were washed three times via centrifugation $(8000 \mathrm{rpm} \times 20 \mathrm{~min})$. Well dispersed GUVs with a size distribution of 8-30 $\mu \mathrm{m}$ were collected $\left(\sim 0.02 \mathrm{mg}\right.$ lipid $\left.\mathrm{mL}^{-1}\right)$ and transferred to a sample chamber for observation.

Immobilization of GUVs for in situ observation. A home-made chamber cell, with a cover-glass substrate, was used for microscope observation in the experiments. For immobilization of the vesicles, the glass substrate was pre-treated with the following procedures. First, the glass slide was washed completely with ethanol and water consequently, boiled in a mixture of $\mathrm{H}_{2} \mathrm{O}_{2}$ and $\mathrm{H}_{2} \mathrm{SO}_{4}$ (3:7 by vol) for $1 \mathrm{~h}$, washed again with a large amount of water followed by drying with $\mathrm{N}_{2}$ flow. It was then dipped into APTES for $5 \mathrm{~min}$, and dried under $\mathrm{N}_{2}$ flow again. After that, the glass slide was kept at $120{ }^{\circ} \mathrm{C}$ for $30 \mathrm{~min}$, before being installed in the chamber for use. For the in-situ microscopy observations, a volume of GUV dispersion was pretransferred to the chamber cell and stabilized for about $5 \mathrm{~min}$ for particle immobilization. After that, certain amount of calcein and peptide solutions were added for the in situ monitoring under confocal microscopy.

Data collection and analysis. During the dynamic entry process of calcein into the interior of a GUV due to drug addition, fluorescence intensity of the GUV interior at each time point (i.e., mean value among pixels read out directly from the Zeiss LSM software) was normalized with that of the surrounding environment and plotted as a function of time.

\section{Interfacial photo-voltage test}

Exponential fitting of the $\mathbf{U} \sim \mathbf{t}$ profile in stage. An exponential function $U=A_{1} e^{-t / \tau}+U_{0}$ was used to fit the membrane action process of each peptide, where $\tau$ stands for the time constant. A time constant of $\tau=42.7 \pm 3.9 \mathrm{~min}, 28.7 \pm 0.5 \mathrm{~min}$, or $2.5 \pm 0.4 \mathrm{~min}$, was obtained for the pure PGLa, pure MAG2, or PGLa/MAG2 mixture system, respectively, although at the same peptide 
concentration of $9 \mu \mathrm{M}$. A time constant of $\tau=15.2 \pm 3.0$ min was obtained for the mixture at a lower concentration of $2.3 \mu \mathrm{M}$. The remarkable difference in $\tau$ clearly demonstrated the different membrane activity of these peptides.

\section{Supplementary images}
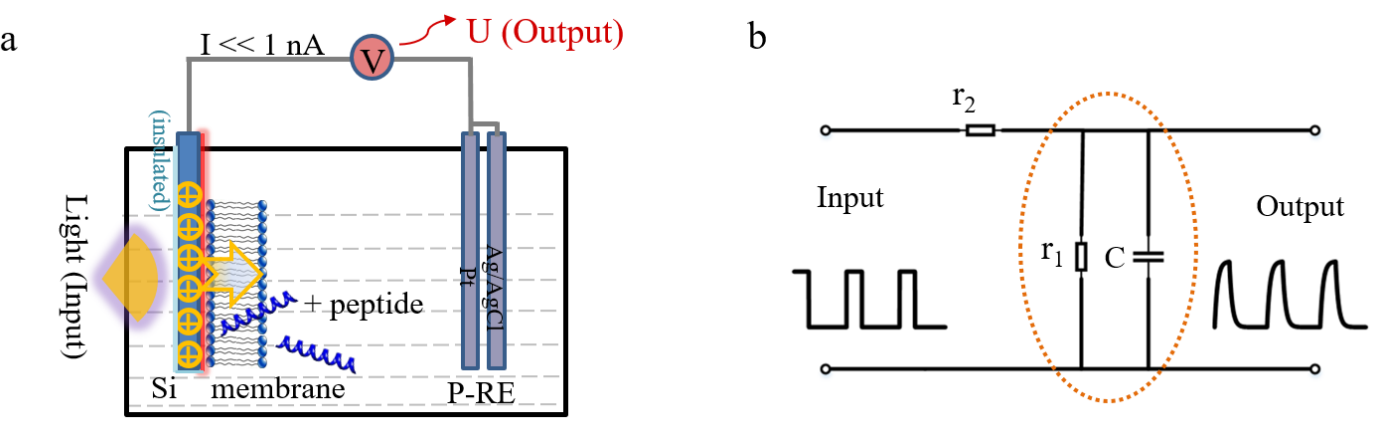

Figure S1. (a) Schematic image showing installations including the working photoelectrode of a silicon wafer deposited with a DOPC membrane, and the reference electrode, both immersed in peptide solution. Square wave modulated illumination for photoelectric generation was used as input and the potential difference (U) was collected as output. (b) Simplified equivalent circuit of the test system. A capacitor $C$ in parallel with a resistor $r_{1}$ (circled in a dotted ellipse) were used to represent the electrical double layer at the solid-liquid interface of the membrane. A schematic equivalent input and output signals were also shown.

Related notes:

For preparation of the working photoelectrode, a lipid membrane was deposited on the pre-cleaned $\mathrm{N}$-doped silicon surface for peptide action, and the area of silicon surface without lipid membrane was entirely covered with transparent insulating glue to avoid any possible leakage of charge from silicon to the environmental solution. During the test, the illumination for photoelectric generation was produced on the glue-side silicon surface as input, and the potential difference (U) was collected simultaneously as output. Therefore, after peptide addition, the time-dependent changes in $U$ value directly reflect changes in electrical double layer at the solid-liquid interface of the membrane, which are determined by changes in structure of the lipid membrane on the silicon surface during membrane-peptide interactions. 
Specifically, it is noted that throughout the measurement, the value of electric current in the circuit keeps less than $1 \mathrm{nA}$. Therefore, the effect of electric signals on peptide actions is minimized, in comparison of that in traditional electrochemical impedance spectroscopy or bilayer capacitance measurements, as the membrane activities of antimicrobial peptides (like PGLa, MAG2, melittin, LL-37, etc) are sensitive to the externally applied voltage due to the electric interactions during their membrane interactions.
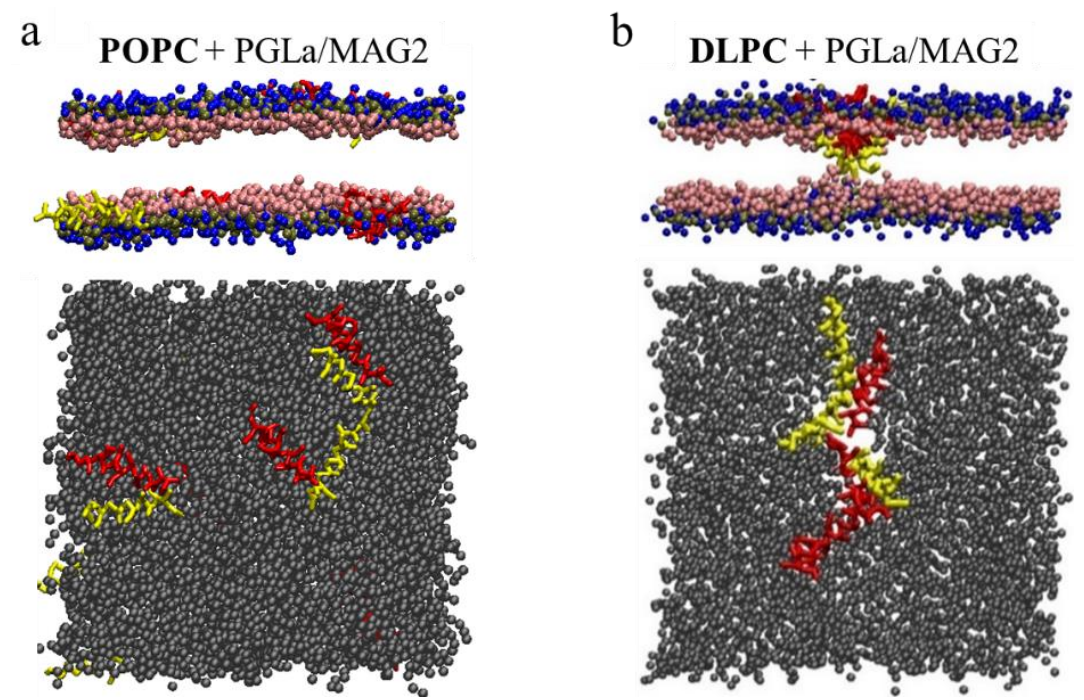

Figure S2. Snapshots showing the interactions of PGLa and MAG2 with (a) POPC lipid bilayer and (b) DLPC bilayer, respectively. Yellow: PGLa; Red: MAG2; Blue, tan and pink: $\mathrm{NC}_{3}, \mathrm{PO}_{4}$ and glycerol beads of lipids. Top: side view, bottom: top view. $\mathrm{P} / \mathrm{L}=1 / 43 ;, N_{P G L a} / N_{M A G 2}=$ 1/1. All snapshots are obtained at $10 \mu \mathrm{s}$. Note that, a membrane pore is spontaneously formed in the DLPC system, while in the POPC system, all peptides still adsorb on the membrane surface during the whole simulations. 


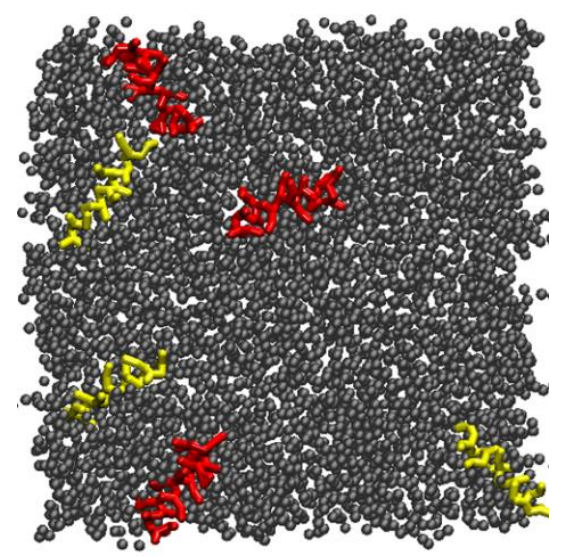

Figure S3. Initial state of peptide-membrane interaction. PGLa (yellow) and MAG2 (red) are randomly distributed on the membrane surface. Lipids are drawn in dark grey for clarification.

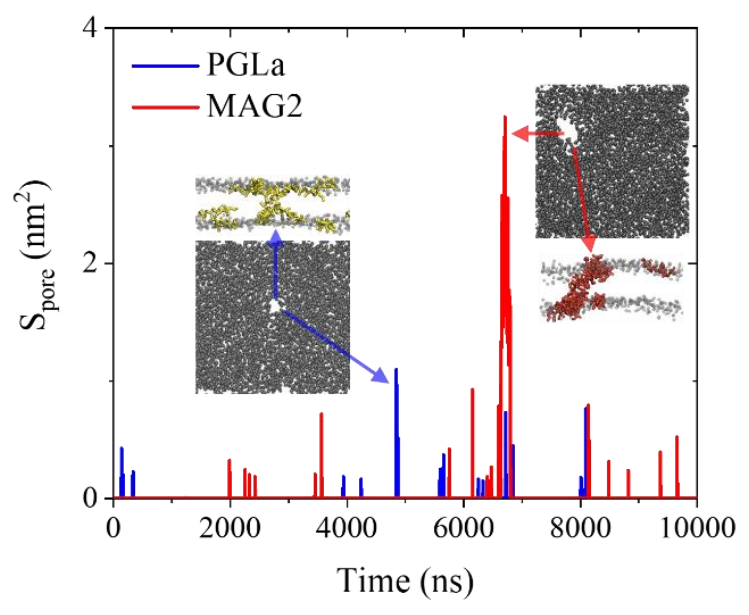

Figure S4. Evolution of pore size under the action of PGLa or MAG2 alone. In both cases, $P / L=$ 1/43. Insets show snapshots of typical transient membrane pores formed by peptides (side view and top view). 
a

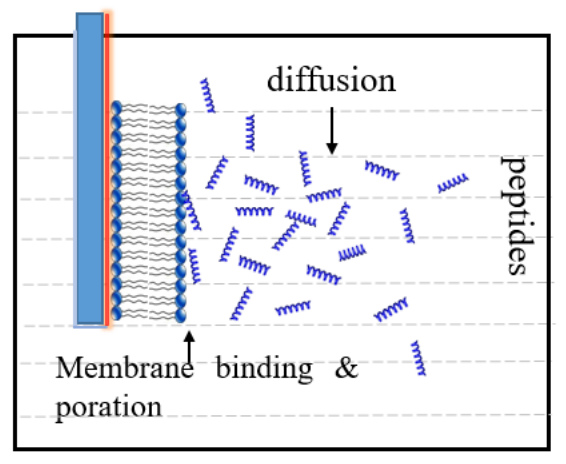

b

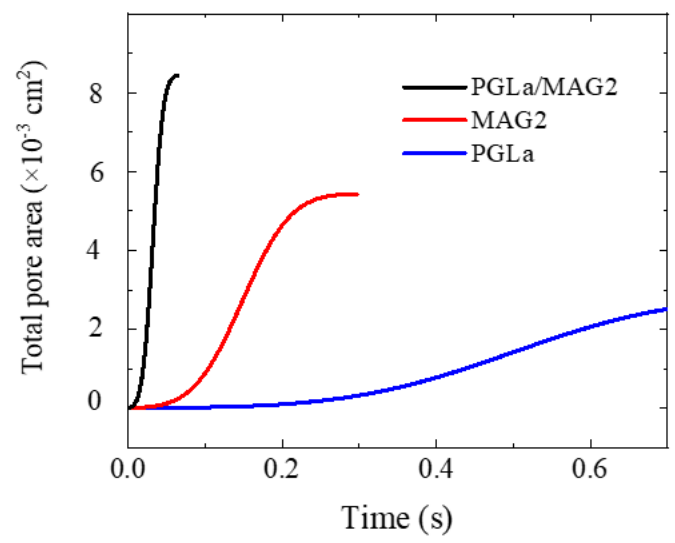

Figure S5. Qualitative analysis of pore formation in the interfacial photo-voltage test. (a) Sketch of the whole interaction process of peptides, including diffusion, membrane binding, and membrane poration. (b) Time evolution of total pore area on membrane due to peptides estimated based on simulation results.

Related notes:

In the experimental tests (e.g., the interfacial photo-voltage test), diffusion of peptide molecules occurs, after addition to the solution and before landing on membrane surface (see Figure S5a). The peptides, with a number of $N \sim 10^{15}$ (at $9 \mu \mathrm{M}$ ), might take a near-Brownian motion in solvent. Thus, at different time interval, the number of peptides arriving at the membrane surface is changing, which should roughly follow a Gaussian distribution (that is, at the initial and final stages, only a small number of peptides reaching the membrane, while in the middle stage, more peptides arriving at the membrane surface).

After landing, the peptides begin to aggregate on the membrane surface and then realize the membrane poration. This should correspond to the simulation process. Assuming the landing peptides are evenly distributed on membrane and the formation of different pores are independent, the probability of occurrence of membrane pores should also obey the Gaussian distribution, as $P(t) \sim e^{-\frac{t^{2}}{\sigma^{2}}}$. However, it is worth noting that, for different peptide systems, the values of $\sigma$ are different. For example, the pores formed by PGLa or MAG2 alone are transient while those produced by PGLa/MAG2 mixture are stable. Thus, for simplification, $\sigma$ is roughly estimated by 
the time required for the increase of unit area of the corresponding pore, as $\sigma_{P / M}=1, \sigma_{M}=4.6$, and $\sigma_{P}=15.6$ (normalized based on the simulation results), for the mixture, pure MAG2, and PGLa, respectively.

Based on these discussions, the total pore area could be calculated as a function of time as $N_{p e p}$. $S_{\text {pep }} \cdot P(t)$. Herein, $N_{\text {pep }}=\alpha \beta N, \alpha$ refers to the ratio of peptides which could land on the membrane surface ( 1\%, estimated by Biochim. Biophys. Acta 2004, 1667, 26) and $\beta$ is the ratio of membrane-bound peptides which could actually take part in pore formation $(\sim 1 \%$, obtained by J. Am. Chem. Soc. 2012, 134, 12732 \& Commun. Theor. Phys. 2019, 71, 887); $S_{p e p}$ is the pore size contributed by each "effective" peptide, which could also be obtained by the simulations, as $S_{P G L a} \sim 0.4 \mathrm{~nm}^{2} /$ peptide, $S_{M A G 2} \sim 0.67 \mathrm{~nm}^{2} /$ peptide, and $S_{p / M} \sim 1.04 \mathrm{~nm}^{2} /$ peptide. Then, time evolution of the total pore area can be obtained as shown in Figure S5b. It is observed that, the PGLa/MAG2 mixture has the fastest growing in pore area while pure PGLa has the slowest one. This result agrees with our experimental observations qualitatively.

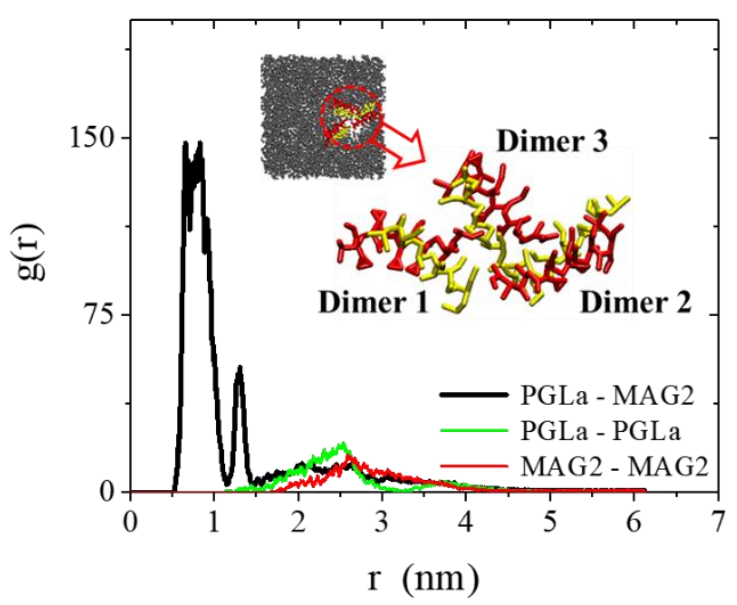

Figure S6. Radial distribution functions of the peptide cluster (inset, snapshot with three heterodimers) in the PGLa/MAG2 mixture. The higher peak of PGLa-MAG2 pair than the others indicates the peptides tended to form PGLa-MAG2 heterodimers rather than PGLa-PGLa or MAG2-MAG2 homodimers. 


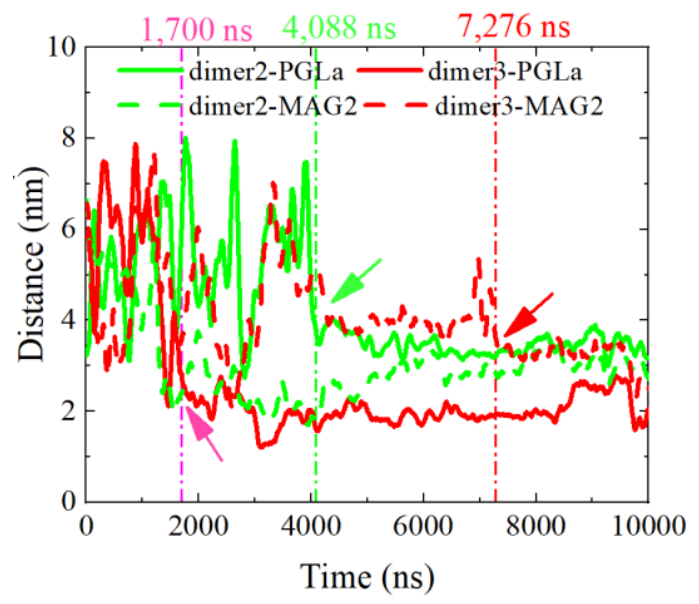

Figure S7. Assembly of peptides from different heterodimers around the first heterodimer, indicated by the distance change between a specific peptide and the first heterodimer. Strong fluctuation in the distance means the random motion of the peptide on the membrane and once it binds to the first heterodimer, the distance between them will decrease and especially keep stable. Thus, all peptides aggregate together finally, as shown in the figure. However, the detailed assembly process is complicated: after the initial random motion, the PGLa (Dimer 3) is recruited by the MAG2 in Dimer 1 (Purple arrow at 1,700 ns), although the MAG2 (Dimer 3) is still far away from them; after a while, the MAG2 (Dimer 2) approaches and binds to Dimer 1, and then the PGLa (Dimer 2) is recruited by this MAG2 to form a heterodimer (Green arrow at 4,088 ns); finally the MAG2 (Dimer 3) arrives and forms a heterodimer with the PGLa (Dimer 3, Red arrow at $7,276 \mathrm{~ns})$.

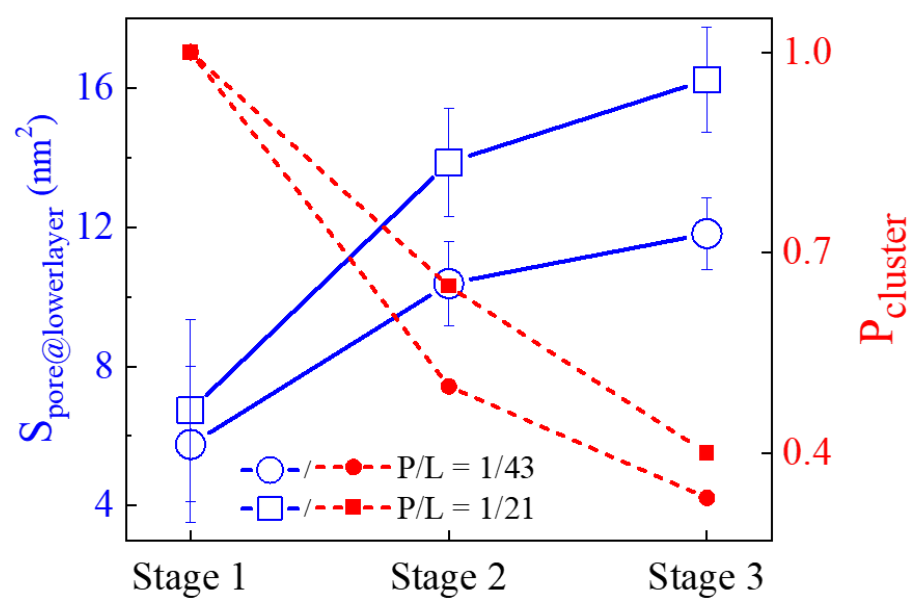


Figure S8. Correlation between the increase of pore size (blue) and contribution of the number of newly-recruited clusters by the pore (Red). $\mathrm{P}_{\text {cluster }}$ is the contribution rate of newly-recruited peptide cluster for the pore growth in each stage, which is defined as the number ratio between the newly-recruited-cluster and the total cluster number in the pore. Stage 1-3 refers to the one in the pore formation shown in Figure 4 (in which marked with different colors). In three stages, the numbers of newly-recruited-cluster are: $1,1,1$ for $P / L=1 / 43$ and $1,2,2$ for $P / L=1 / 21$.

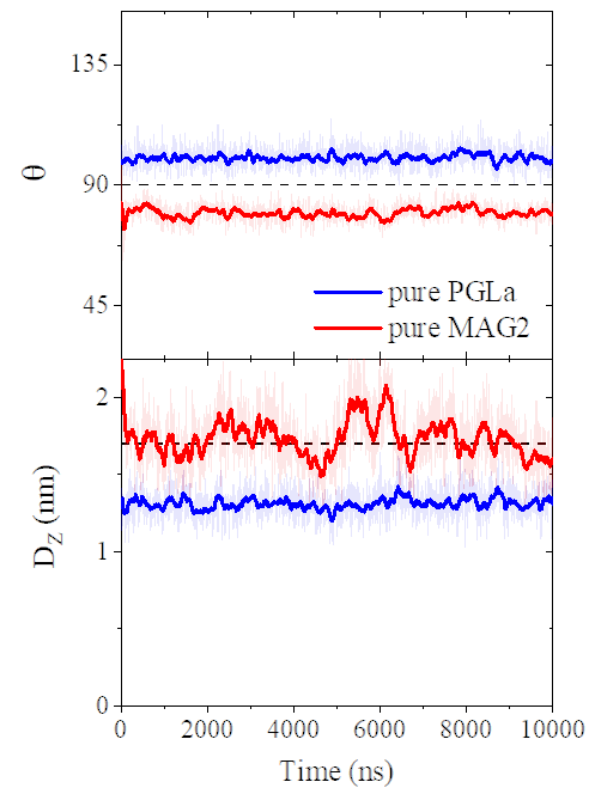

Figure S9. Time-dependent evolutions of $\theta$ and $D_{Z}$ of pure PGLa and MAG2 during the membrane interaction process. 

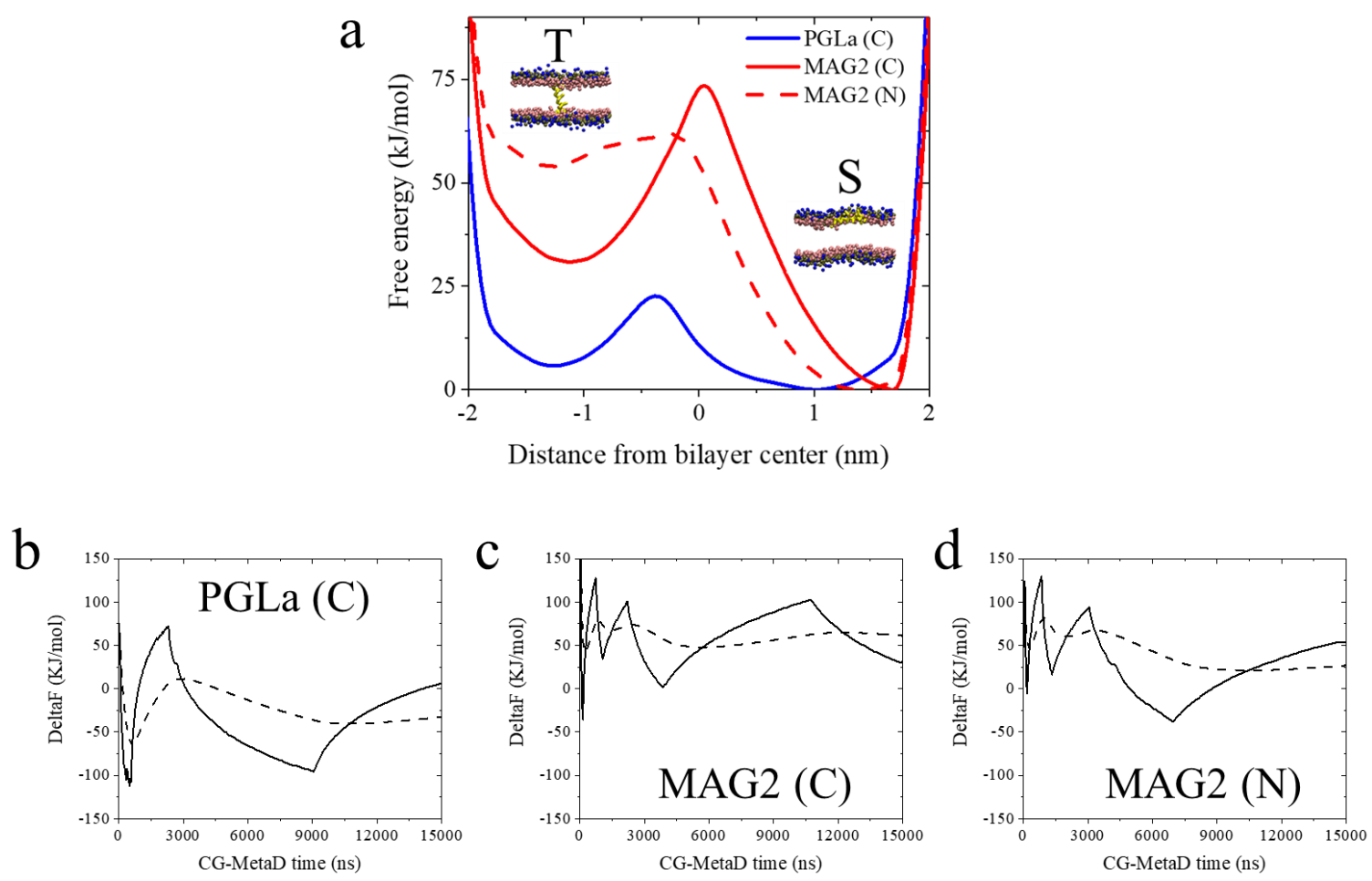

Figure S10. (a) Free energy distribution of a PGLa or MAG2 peptide reorientating from membrane-binding state (State S) to transmembrane insertion state (State T), with its C- or Nterminus. (b-d) Convergence of the free energy of the transmembrane penetration of a peptide. The black line refers to the free energy difference between the membrane binding state and the transmembrane insertion state, and the dashed line shows the progressive average of the free energy difference.

\section{SUPPLEMENTARY TABLE}

Table S1. Influence of $P / L$ ratios on the peptide-membrane interactions. All snapshots (side view) are obtained at $\mathrm{t}=10 \mu \mathrm{s}$. Pores are highlighted with green dash circles. 


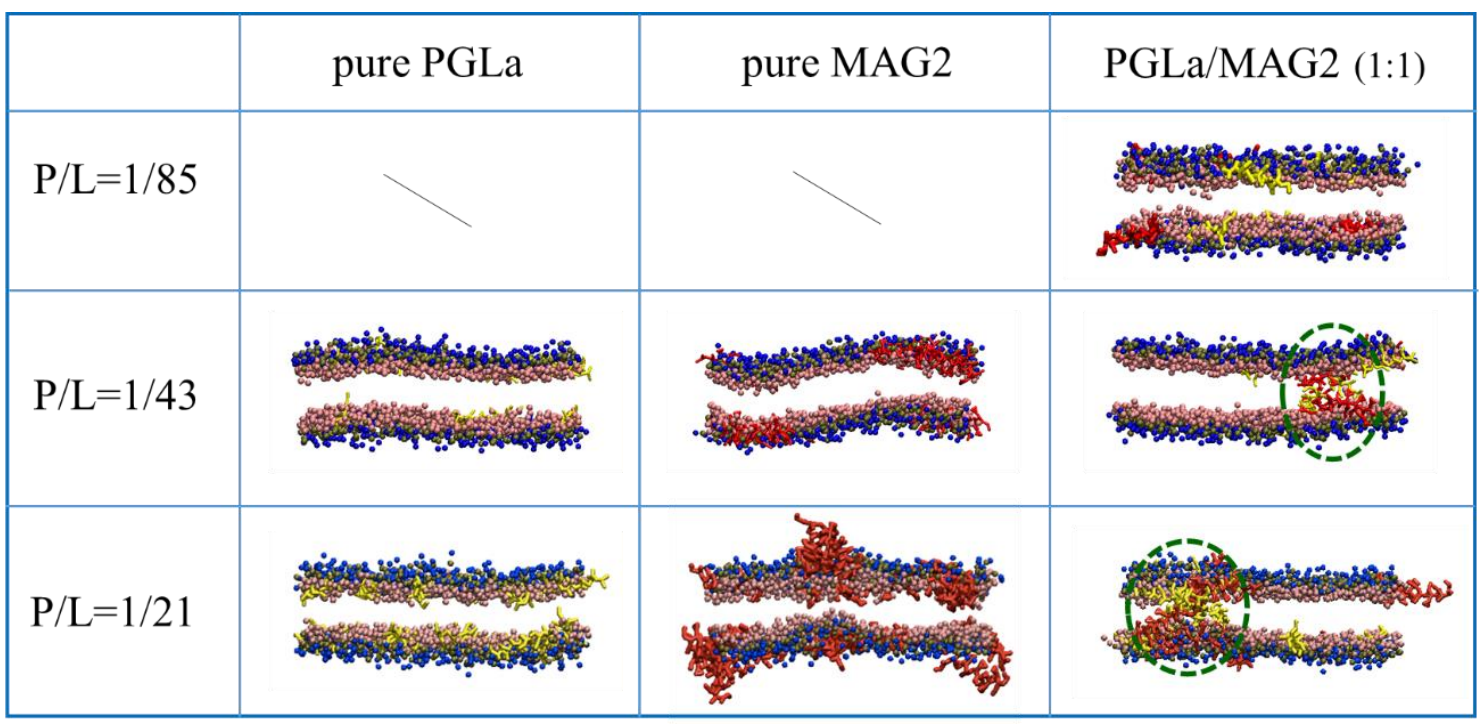

